TAIWANESE JOURNAL OF MATHEMATICS

Vol. 12, No. 3, pp. 623-633, June 2008

This paper is available online at http://www.tjm.nsysu.edu.tw/

\title{
OSCILLATION THEOREM FOR SECOND-ORDER DIFFERENCE EQUATIONS
}

\author{
Jinfa Cheng and Yuming Chu
}

\begin{abstract}
Sufficient and necessary conditions are established for the secondorder difference equations

$$
\Delta\left(r_{n-1} \Delta x_{n-1}\right)+p_{n} x_{n}^{\gamma}=0, n=1,2, \ldots
$$

where $\gamma$ is the quotient of odd positive integers. Our results extend the well known oscillation theorem which was proved in [1,JMAA,91:9-29,1983], and answer an open problem in [2] when $r_{n}=1, \gamma=1$.
\end{abstract}

\section{INTRODUCTION}

Consider the second order difference equations

$$
\Delta\left(r_{n-1} \Delta x_{n-1}\right)+p_{n} x_{n}^{\gamma}=0, n=1,2, \ldots
$$

where $\Delta x_{n}=x_{n+1}-x_{n}, \gamma$ is the quotient of odd positive integers and $p_{n}, r_{n} \in$ $(0, \infty)$ for $n=1,2, \ldots$ with $p_{n}$ not eventually equal to zero. Denote

$$
R_{n}=\sum_{s=0}^{n-1} \frac{1}{r_{s}}
$$

and assume

$$
\lim _{n \rightarrow \infty} R_{n}=\sum_{s=0}^{\infty} \frac{1}{r_{s}}=\infty .
$$

Received March 4, 2006, accepted June 30, 2006.

Communicated by Sze-Bi Hsu.

2000 Mathematics Subject Classification: 39A11.

Key words and phrases: Oscillation, Nonoscillatory solution, Difference equations, Contraction principle.

This research is supported by NNSF of P. R. China (No. 10601040) and 973 project of P. R. China (No. 2006CB708304). 
A solution $x_{n}$ of Eq. (1) is oscillatory if $x_{n}$ are neither eventually all positive nor eventually all negative. Otherwise, it is called nonoscillatory.

Eq. (1) is a discrete analogue of the second order equation

$$
\left(r(t) y^{\prime}\right)^{\prime}+p(t) y^{\gamma}=0
$$

which appears in astrophysics, relativistic mechanics,nuclear physics,chemical reactions, etc (See [2]).

we say that Eq. (1) and Eq.(4) is strictly superlinear if $\gamma>1$; strictly sublinear if $0<\gamma<1$; and linear if $\gamma=1$.

When $r_{n}=1$,Eq. (1) was reduced to

$$
\Delta^{2} x_{n-1}+p_{n} x_{n}^{\gamma}=0, n=1,2, \ldots
$$

which is the discrete analogue of the Emden-Fowler equation ([2])

$$
y^{\prime \prime}+p(t) y^{\gamma}=0 .
$$

In the past years, the theory of the oscillatory behavior of second-order differential and difference equations have been investigated by many authors, and numerous oscillation criteria have been obtained (see [1-9]). For example, for the linear differential equations, that is, when $\gamma=1$, numerous oscillation criteria have been obtained. when $\gamma=1, r(t)=1$, most important conditions that guarantee Eq.(4) is oscillatory as follows:

(A1) $\lim _{t \rightarrow \infty} \int_{t_{0}}^{t} p(s) d s=\infty . \quad$ (Fite $\left.[3]\right)$

(A2) $\lim _{t \rightarrow \infty} \frac{1}{t} \int_{t_{0}}^{t} \int_{t_{0}}^{s} p(u) d u d s=\infty . \quad($ Wintner $[4])$

(A3) $-\infty<\lim _{t \rightarrow \infty} \inf \frac{1}{t} \int_{t_{0}}^{t} \int_{t_{0}}^{s} p(u) d u d s<\lim _{x \rightarrow \infty} \sup \frac{1}{t} \int_{t_{0}}^{t} \int_{t_{0}}^{s} p(u) d u d s \leq \infty$. (Hart$\operatorname{man}[5])$.

Kamenev [6] gave another condition for the oscillation, i.e.,

(A4) $\lim _{t \rightarrow \infty} \sup \frac{1}{t^{n}} \int_{0}^{t}(t-s)^{n} p(s) d s=\infty, n>1$.

The Kamenev criterion has been extended by several authors. Among them, when $\gamma=1, r(t) \neq 1$, Philos [7], Q.Kong [8] obtain results on the oscillation by replacing the kerned function $(t-s)^{n}$ by a general class of functions $H(t, s)$ satisfying certain assumptions. This class of functions is as follows: Let $H: D \equiv$ $\left\{(t, s): t \geq s \geq t_{0}\right\} \rightarrow R$ be a continuous function such that

$$
H(t, t)=0 \text { for } t \geq t_{0} \text { and } H(t, s)>0 \text { for } t>s \geq t_{0},
$$

and has a continuous and nonpositive partial derivative $H_{s}(t, s)$ on $D$ with respect to the second variable. Moreover, let $h: D \rightarrow R$ be a continuous function with

$$
H_{s}(t, s)=-h(t, s) \sqrt{H(t, s)} \quad \text { for } \quad \text { all } \quad(t, s) \in D,
$$


some related concrete results can be found in [7-8] and the references therein. However, most of these articles only discussed the sufficient conditions by use of the Raccati technique and integral averaging technique. Just a few of them have studied the necessary and sufficient conditions for the oscillatory behavior. In [1], the authors studied oscillatory criteria for Eq.(5) and obtained very important results. To the best of our knowledge, paper [1] is probably the only one publications on the necessary and sufficient conditions for the oscillatory of solution of second-order difference equations.

The following theorem was established by Hooker and Patula [1].

Theorem A. (a) Assume that

$$
\sum_{n=1}^{\infty} n p_{n}<\infty
$$

Then Eq. (5) has a bounded nonoscillatory solution.

(b) Assume that $\gamma>1$. Then every solution of Eq. (5) oscillates if and only if

$$
\sum_{n=1}^{\infty} n p_{n}=\infty
$$

(c) Assume that $\gamma=1$ and

$$
\sum_{n=1}^{\infty} p_{n}=\infty .
$$

Then every solution of Eq. (5) oscillates.

(d) Assume that $0<\gamma<1$, then every solution of Eq. (5) oscillates if and only if

$$
\sum_{n=1}^{\infty} n^{\gamma} p_{n}=\infty
$$

It is interesting to note that when $\gamma \neq 1$, that is when Eq.(5) is nonlinear, we know necessary and sufficient conditions for oscillation. However, for the linear equation, that is when $\gamma=1$, necessary and sufficient conditions for oscillation are not known. It follows from Theorem A (a) that when $\gamma=1$,

$$
\sum_{n=1}^{\infty} n p_{n}=\infty
$$

is a necessary condition for all bounded solutions to oscillate. But is it sufficient? (see[2]) 
Motived by these results, our aim in this paper is to investigate the oscillatory of the solution of so-called generalized Emden-Fowler difference equation (1) and establish several sufficient and necessary conditions. Our following results extent Theorem A and answer the above problem .

Our main results are:

Theorem. Assume that (3) holds, then the following statements are true:

(a) Assume that

$$
\sum_{n=1}^{\infty} R_{n} p_{n}<\infty
$$

Then Eq. (1) has a bounded nonoscillatory solution.

(b) Assume that $\gamma>1$, then every solution of Eq. (1) oscillates if and only if

$$
\sum_{n=1}^{\infty} R_{n} p_{n}=\infty .
$$

(c) Assume that $\gamma=1$, then every bounded solution of Eq. (1) oscillates if and only if

$$
\sum_{n=1}^{\infty} R_{n} p_{n}=\infty
$$

(d) Assume that $0<\gamma<1$, then every solution of Eq. (1) oscillates if and only if

$$
\sum_{n=1}^{\infty} R_{n}^{\gamma} p_{n}=\infty
$$

Remark 1. When $r_{n}=1$, then $R_{n}=n$, our theorem extend and improve Theorem A.

\section{The Proof of the Theorem}

Before we present the proof of the theorem we need the following lemma.

Lemma. Assume that (3) holds and the Eq. (1) has nonoscillatory solution. Then Eq. (1) has a solution $\left\{x_{n}\right\}$ such that for some $N>0$,

$$
x_{n}>0, \Delta x_{n}>0, \text { and } \quad \Delta\left(r_{n} \Delta x_{n}\right)<0 \quad \text { for } \quad n \geq N .
$$


Proof. As $\gamma$ is the quotient of odd integers, the opposite of a solution is also a solution and so Eq. (1) has a solution $\left\{x_{n}\right\}$ which is eventually positive. That is, there exists $N>0$ such that

$$
x_{n}>0 \quad \text { for } \quad n \geq N \text {. }
$$

From Eq. (1) we see that

$$
\Delta\left(r_{n-1} \Delta x_{n-1}\right)=-p_{n} x_{n}^{\gamma}<0, \quad \text { for } \quad n \geq N
$$

Therefore, $r_{n-1} \Delta x_{n-1}$ is monotone decreasing for $n \geq N$. The next cases are possible:

Case 1. $r_{n-1} \Delta x_{n-1}<0, n \geq N$. then we have $r_{n-1} \Delta x_{n-1} \leq r_{N-1} \Delta x_{N-1}=$ $c_{N-1}<0, n \geq N$, and obtain $\Delta x_{n-1} \leq c_{N-1} \frac{1}{r_{n-1}}, x_{n} \leq x_{N-1}+c_{N-1} \sum_{s=N}^{n} \frac{1}{r_{s-1}} \rightarrow$ $-\infty$, which contradicts the assumption that $x_{n}>0$.

Case 2. $r_{n-1} \Delta x_{n-1}>0, n \geq N$. In this case, we have

$$
\Delta x_{n}>0, n \geq N
$$

The proof of the lemma is complete.

Proof of the Theorem.

(a) Assume that (11) holds. We must prove that Eq. (1) has a bounded nonoscillatory solution.

Observe that if $\left\{x_{n}\right\}$ satifies the equation

$$
x_{n}=1-\sum_{i=n+1}^{\infty}\left(R_{i}-R_{n}\right) p_{i} x_{i}^{\gamma},
$$

then $\left\{x_{n}\right\}$ is a solution of Eq. (1). Therefore it suffices to show that Eq. (16) has bounded nonoscillatory solution. To this end, choose $N$ so large that

$$
\max \left\{\sum_{i=N}^{\infty} R_{i} p_{i}, 2 \gamma \sum_{i=N}^{\infty} R_{i} p_{i}\right\}<\frac{1}{2} .
$$

Consider the Banach space $l_{\infty}^{N}$ of all bounded, real sequences $z=\left\{z_{n}\right\}_{n \geq N}$ with the norm defined by $\|z\|=\sup _{n \geq N}\left|z_{n}\right|$. 
Set

$$
S=\left\{z \in l_{\infty}^{N}: \frac{1}{2} \leq z_{n} \leq 1, n \geq N\right\} .
$$

Clearly $S$ is a closed subset of $l_{\infty}^{N}$. Define the mapping $T$ on $S$ by

$$
(T z)_{n}=1-\sum_{i=n+1}^{\infty}\left(R_{i}-R_{n}\right) p_{i} z_{i}^{\gamma} \quad \text { for } n \geq N .
$$

Note that $z_{i}^{\gamma} \leq 1$ and so

$$
(T z)_{n} \geq 1-\sum_{i=n+1}^{\infty}\left(R_{i}-R_{n}\right) p_{i} \geq \frac{1}{2} \quad \text { for } n \geq N .
$$

Also clearly, $(T z)_{n} \leq 1$. Thus $T: S \rightarrow S$. We now claim that $T$ is a contraction on $S$. Set $f(x)=x^{\gamma}$, we find for $x_{1}, x_{2} \in\left(\frac{1}{2}, 1\right)$,

$$
\left|x_{1}^{\gamma}-x_{2}^{\gamma}\right| \leq\left|f^{\prime}(\xi)\right|\left|x_{1}-x_{2}\right|, \text { where } \xi \in\left(\min \left\{x_{1}, x_{2}\right\}, \max \left\{x_{1}, x_{2}\right\}\right) .
$$

But

$$
|f(x)|=\left|\gamma \xi^{\gamma-1}\right| \leq \begin{cases}\gamma & \text { if } \gamma \geq 1 \\ 2 \gamma & \text { if } 0<\gamma<1\end{cases}
$$

And so

$$
\left|x_{1}^{\gamma}-x_{2}^{\gamma}\right| \leq 2 \gamma\left|x_{1}-x_{2}\right| \quad \text { for } x_{1}, x_{2} \in\left(\frac{1}{2}, 1\right) .
$$

Let $z, w \in S$. Then for $n \geq N$,

$$
\begin{aligned}
\left|(T z)_{n}-(T w)_{n}\right| & \leq \sum_{i=n+1}^{\infty}\left(R_{i}-R_{n}\right) p_{i}\left|z_{i}^{\gamma}-w_{i}^{\gamma}\right| \\
& \leq 2 \gamma \sum_{i=n+1}^{\infty}\left(R_{i}-R_{n}\right) p_{i}\left|z_{i}-w_{i}\right| \\
& \leq 2 \gamma|z-w| \sum_{i=n+1}^{\infty}\left(R_{i}-R_{n}\right) p_{i} \leq \frac{1}{2}|| z-w \| .
\end{aligned}
$$

Hence

$$
\|T z-T w\| \leq \frac{1}{2}\|z-w\| .
$$

and so $T$ is a contraction on $S$. The (unique) fixed point of $T$ is the desired bounded, nonoscillatory solution of Eq. (16). 
(b) Assume that (12) holds,we must prove every solution of Eq. (1) oscillates. Otherwise by lemma, Eq. (1) has a solution $\left\{x_{n}\right\}$ such that (15) holds. By multiplying both sides of Eq. (1) by $R_{n} x_{n}^{-\gamma}$ and then by summing up we obtain

$$
\sum_{n=N}^{k-1} R_{n} x_{n}^{-\gamma} \Delta\left(r_{n-1} \Delta x_{n-1}\right)+\sum_{n=N}^{k-1} R_{n} p_{n}=0 .
$$

By using the summation-by-parts formula we find

$$
R_{k} x_{k}^{-\gamma} r_{k-1} \Delta x_{k-1}-R_{N} x_{N}^{-\gamma} r_{N-1} \Delta x_{N-1}-\sum_{n=N}^{k-1} r_{n} \Delta x_{n} \Delta\left(R_{n} x_{n}^{-\gamma}\right)+\sum_{n=N}^{k-1} R_{n} p_{n}=0 .
$$

Hence

$$
\sum_{n=N}^{k-1} r_{n} \Delta x_{n} \Delta\left(R_{n} x_{n}^{-\gamma}\right)=\infty
$$

Now observe that

$$
\Delta\left(R_{n} x_{n}^{-\gamma}\right)=\frac{1}{r_{n}} x_{n+1}^{-\gamma}+R_{n} \Delta\left(x_{n}^{-\gamma}\right) \leq \frac{1}{r_{n}} x_{n+1}^{-\gamma} .
$$

and so

$$
\sum_{n=N}^{k-1} r_{n} \Delta x_{n} \Delta\left(R_{n} x_{n}^{-\gamma}\right) \leq \sum_{n=N}^{k-1} r_{n} \Delta x_{n} \frac{1}{r_{n}} x_{n+1}^{-\gamma}=\sum_{n=N}^{k-1} \Delta x_{n} x_{n+1}^{-\gamma}
$$

Set $f(x)=x_{n}+\left(\Delta x_{n}\right)(x-n)$ for $n \leq x \leq n+1$ and $n \geq N$, then $f$ is continuous and increasing for $x \geq N$, and so

$$
\begin{aligned}
x_{n+1}^{-\gamma} \Delta x_{n} & =\int_{n}^{n+1} x_{n+1}^{-\gamma} \Delta x_{n} d x=\int_{n}^{n+1} f(n+1)^{-\gamma} f^{\prime}(x) d x \\
& <\int_{n}^{n+1} f(x)^{-\gamma} f^{\prime}(x) d x=\frac{1}{\gamma-1}\left[f(n+1)^{1-\gamma}-f(n)^{1-\gamma}\right]
\end{aligned}
$$

By summing up from $n=N$ to $n=k-1$ we obtain

$$
\sum_{n=N}^{k-1} x_{n+1}^{-\gamma} \Delta x_{n} \leq \frac{1}{\gamma-1}\left[f(k)^{1-\gamma}-f(N)^{1-\gamma}\right] \leq \frac{f(N)^{1-\gamma}}{\gamma-1}
$$

which because of (22) contradicts (21).

Conversely we must prove that if every solution of Eq. (1) oscillates and $\gamma>1$, then (12) holds. Otherwise (11) holds and by (a) we obtain the contraction that Eq. (1) has a nonoscillatory solution. 
(c) Assume that $\gamma=1$, We will prove that every bounded solution of Eq. (1) oscillates. Otherwise by lemma, Eq. (1) has a solution $\left\{x_{n}\right\}$ such that (15) holds, then $\lim _{n \rightarrow \infty} x_{n}=c>0$, and there exists $N>0$ such that

$$
\frac{c}{2} \leq x_{n} \leq c, \quad \text { for } \quad n>N .
$$

By means of Eq. (1), we have

$$
\Delta\left(r_{n-1} \Delta x_{n-1}\right)+\frac{c}{2} p_{n} \leq 0, \text { for } \quad n>N,
$$

multiplying both sides of inequality (23) by $R_{n}$ and then by summing up we obtain

$$
\sum_{n=N}^{k-1} R_{n} \Delta\left(r_{n-1} \Delta x_{n-1}\right)+\frac{c}{2} \sum_{n=N}^{k-1} R_{n} p_{n} \leq 0 .
$$

By the summation-by-parts formula we have

$$
R_{k} r_{k-1} \Delta x_{k-1}-R_{N} r_{N-1} \Delta x_{N-1}-\sum_{n=N}^{k-1} r_{n} \Delta x_{n} \Delta R_{n}+\frac{c}{2} \sum_{n=N}^{k-1} R_{n} p_{n} \leq 0
$$

Observe that $\sum_{n=N}^{k-1} r_{n} \Delta x_{n} \Delta R_{n}=\sum_{n=N}^{k-1} \Delta x_{n}=x_{k}-x_{N}$ then we get

$$
R_{k} r_{k-1} \Delta x_{k-1}-R_{N} r_{N-1} \Delta x_{N-1}-x_{k}+x_{N}+\frac{c}{2} \sum_{n=N}^{k-1} R_{n} p_{n} \leq 0,
$$

from above inequality we have $x_{k} \rightarrow \infty$, which obviously contradicts the bounded behavior of $x_{n}$.

Conversely, we should prove that if every bounded solution of Eq. (1) oscillates then (13) holds. Otherwise (11) holds and by (a) we obtain the contraction that Eq. (1) has a bounded nonoscillatory solution.

(d) Assume that (14) holds, we shall prove that every solution of Eq. (1) oscillates. Otherwise by lemma, Eq. (1) has a solution $\left\{x_{n}\right\}$ such that (15) holds.

Set $g_{n}=r_{n} \Delta x_{n}$, then $\Delta g_{n}=\Delta\left(r_{n} \Delta x_{n}\right)<0, g_{n}$ is decreasing for $n \geq N$. Observe that for $n \geq N$,

$$
x_{n}-x_{N}=\sum_{s=N}^{n-1} \Delta x_{s}=\sum_{s=N}^{n-1} \frac{g_{s}}{r_{s}} \geq g_{n} \sum_{s=N}^{n-1} \frac{1}{r_{s}}
$$

and so

$$
\frac{x_{n}}{g_{n}}=\frac{x_{n}}{r_{n} \Delta x_{n}} \geq \sum_{s=N}^{n-1} \frac{1}{r_{s}}, \quad \text { for } \quad n \geq N
$$


By dividing both terms of Eq. (1) by $\left(r_{n} \Delta x_{n}\right)^{\gamma}$, and then applying (24), and finally by summing up from $n=N$ to $n=k$, we obtain

$$
\sum_{n=N}^{k} \frac{\Delta\left(r_{n} \Delta x_{n}\right)}{\left(r_{n} \Delta x_{n}\right)^{\gamma}}+\sum_{n=N}^{k} R_{n}^{\gamma} p_{n} \leq 0 .
$$

In view of (25) it follows that

$$
\sum_{n=N}^{k} \frac{\Delta\left(r_{n} \Delta x_{n}\right)}{\left(r_{n} \Delta x_{n}\right)^{\gamma}}=-\infty
$$

Set $g(x)=r_{n} \Delta x_{n}+\Delta\left(r_{n} \Delta x_{n}\right)(x-n) \quad$ for $\quad n \leq x \leq n+1$ and $n \geq N$, then $g(x)$ is continuous and decreasing for $n \geq N$.

$$
g(x) \leq g(n)=r_{n} \Delta x_{n}, \quad \text { for } \quad n \leq x \leq n+1 .
$$

Then we have

$$
\frac{\Delta\left(r_{n} \Delta x_{n}\right)}{\left(r_{n} \Delta x_{n}\right)^{\gamma}}=\int_{n}^{n+1} \frac{\Delta\left(r_{n} \Delta x_{n}\right)}{\left(r_{n} \Delta x_{n}\right)^{\gamma}} d x \geq \int_{n}^{n+1} \frac{g^{\prime}(x)}{g^{\gamma}(x)} d x
$$

By summing up from $n=N$ to $n=k$, this implies that

$$
\sum_{n=N}^{k} \frac{\Delta\left(r_{n} \Delta x_{n}\right)}{\left(r_{n} \Delta x_{n}\right)^{\gamma}} \geq \frac{g^{1-\gamma}(k)-g^{1-\gamma}(N)}{1-\gamma} \geq \frac{-g^{1-\gamma}(N)}{1-\gamma},
$$

which, as $k \rightarrow \infty$ contradicts (26).

Conversely, we should prove that if every solution of Eq. (1) oscillates, then (14) holds. Otherwise $\sum_{n=1}^{\infty} R_{n}^{\gamma} p_{n}<\infty$.

Now choose $N_{0}$ so large that $\sum_{n=N_{0}}^{\infty} R_{n}^{\gamma} p_{n}<\frac{1}{2}$. Let $\left\{x_{n}\right\}$ be the unique solution of solution of Eq. (1) with

$$
x_{N_{0}}=0, x_{N_{0}+1}=\frac{1}{r_{N_{0}}} .
$$

That is

$$
x_{N_{0}}=0, g_{N_{0}}=r_{N_{0}} \Delta x_{N_{0}}=1 .
$$

By induction, we can prove that

$$
\frac{1}{2} \leq g_{n}=r_{n} \Delta x_{n} \leq 1 \text { for all } n \geq N_{0}
$$


In fact, assume that $\frac{1}{2} \leq r_{n} \Delta x_{n} \leq 1$ for $n \leq N-1$,where $N>N_{0}$, then it is obvious that $x_{n} \geq 0$ for $n \leq N-1$. We obtain

$$
x_{n}=x_{n}-x_{N_{0}}=\sum_{s=N_{0}}^{n-1} \Delta x_{s}=\sum_{s=N_{0}}^{n-1} \frac{g_{s}}{r_{s}} \leq \sum_{s=N_{0}}^{n-1} \frac{1}{r_{s}} \quad \text { for } \quad n \leq N .
$$

Therefore

$$
\begin{aligned}
1 \geq r_{N} \Delta x_{N} & =r_{N_{0}} \Delta x_{N_{0}}-\sum_{s=N_{0}}^{N-1} p_{s} x_{s}^{\gamma} \\
& \geq r_{N_{0}} \Delta x_{N_{0}}-\sum_{s=N_{0}}^{\infty} p_{s} R_{s}^{\gamma} \geq 1-\frac{1}{2}=\frac{1}{2} .
\end{aligned}
$$

That is: $\frac{1}{2} \leq r_{n} \Delta x_{n} \leq 1$ for $n=N$. So (27) holds. $\left\{x_{n}\right\}$ is a nonoscillatory solution of Eq. (1). This contradiction completes the proof of part (d).

The proof of the theorem is complete.

Remark 2. From the above proof of the theorem, we see that our results are also valid for following more general equation:

$$
\Delta\left(r_{n-1} \Delta x_{n-1}\right)+p_{n}\left|x_{n}\right|^{\gamma} \operatorname{sgn} x_{n}=0, n=1,2, \ldots
$$

where $\gamma>0$.

\section{ACKNOWLEDGMENT}

We would like to thank Prof. A.B.Dishliev for his valuable advices and very careful reading of the manuscript. We also thank the referee for his many valuable suggestions on our paper.

\section{REFERENCES}

1. J. W. Hooker, and W. T. Patula, A second order nonlinear difference equation: Oscillation and asymptotic growth. J. Math. Anal. Appl., 91 (1983), 9-29.

2. V. L. Kocic, and G. E. Ladas, Global Behavior of Nonlinear Difference Equations of Higher Order with Applications, Kluwer Academic Publishers, 1993.

3. W. B. Fite, Concerning the zeros of the solutions of certain differential equations, Trans. Amer. Math. Soc., 19 (1918), 341-352.

4. A. Wintner, A criterion of oscillatory, Quart. Appl. Math., 7 (1949), 115-117,. 
5. P. Hartman, On nonoscillatory linear differential equations of second, Amer. J. Math., 74 (1952), 389-400.

6. I. V. Kamenev, An integral criterion for oscillation of linear differential equations of second order (in Russian), Mat. Zametki, 23 (1978), 249-251.

7. C. G. Philos, Oscillation criteria for second order superlinear differential equations, Canad. J. Math., 41 (1989), 321-340.

8. Q. Kong, Interval criteria for oscillation of second-order linear ordinary differential equations, J. Math. Anal. Appl., 229 (1999), 258-270.

9. R. P. Agarwal, S. R. Grace and D. O'Regan, Oscillation theory for difference and functional differential equations, Kluwer, Dordrecht, 2000.

\author{
Jinfa Cheng \\ Department of Mathematics, \\ Xiamen University, \\ Xiamen 361005, \\ P. R. China \\ E-mail: jfcheng@xmu.edu.cn \\ Yuming Chu \\ Department of Mathematics, \\ Huzhou Teacher's College, \\ Huzhou 313000, \\ P. R. China \\ E-mail: chuyuming@hutc.zj.cn
}

